\title{
Comparative transcriptome analysis of mulberry reveals anthocyanin biosynthesis mechanisms in black (Morus atropurpurea Roxb.) and white (Morus alba L.) fruit genotypes
}

Gaiqun Huang ${ }^{1,2+}$, Yichun Zeng ${ }^{2 \dagger}$, Ling Wei ${ }^{2}$, Yongquan Yao ${ }^{2}$, Jie Dai ${ }^{2}$, Gang Liu ${ }^{2^{*}}$ and Zhongzheng Gui ${ }^{1,3^{*}}$ (D)

\begin{abstract}
Background: To gain a better understanding of anthocyanin biosynthesis in mulberry fruit, we analyzed the transcriptome of the mulberry varieties Da 10 (Morus atropurpurea Roxb., black fruit) and Baisang (Morus alba L., white fruit).

Results: We found that whereas Da 10 had high levels of cyanidin 3-O-glucoside (Cy), and pelargonidin 3-Oglucoside (Pg), Baisang contained only Cy, at low levels. Based on a comparative transcriptome analysis, we annotated more than 27,085 genes (including 1735 new genes). Genes that were differentially expressed between Da 10 and Baisang were detected at three stages of fruit development: S1 [4256 genes, 10 days post-anthesis (DPA)], S2 (5612 genes, 19 DPA), and S3 (5226 genes, 28 DPA). Anthocyanin biosynthesis was found to be associated with the expression of 15 core genes and 5 transcription factors. Relative to Baisang, Da 10 showed a significant upregulation of genes involved in the early stages (production of the intermediate compounds chalcone and dihydroflavonol) and late stages (production of Cy and Pg) of anthocyanin biosynthesis. Baisang showed a significant downregulation of the genes involved in the early stages of anthocyanin biosynthesis and overexpression of flavanone 3-hydroxylase (FLS), resulting in the generation of quercetin and/or myricetin but not anthocyanins.

Conclusions: The biosynthesis of anthocyanin in mulberry fruit is initiated from the precursor, phenylalanine, and mediated by the upregulation of dihydroflavonol 4-reductase, anthocyanidin synthase, anthocyanidin 3-Oglucosyltransferase, and cyanidin-3-O-glucoside 2-O-glucuronosyltransferase, and downregulation of FLS to produce Cy and Pg.
\end{abstract}

Keywords: Mulberry fruit, Anthocyanin, Biosynthesis, Transcriptome

\footnotetext{
*Correspondence: liugang2004380@sina.com; srizzgui@hotmail.com

${ }^{\dagger}$ Gaiqun Huang and Yichun Zeng contributed equally to this work.

${ }^{2}$ Sericultural Research Institute, Sichuan Academy of Agricultural Sciences, Nanchong 637000, Sichuan, China

${ }^{1}$ School of Biotechnology, Jiangsu University of Science and Technology, Zhenjiang 212018, Jiangsu, China

Full list of author information is available at the end of the article
}

(C) The Author(s). 2020 Open Access This article is licensed under a Creative Commons Attribution 4.0 International License, which permits use, sharing, adaptation, distribution and reproduction in any medium or format, as long as you give appropriate credit to the original author(s) and the source, provide a link to the Creative Commons licence, and indicate if changes were made. The images or other third party material in this article are included in the article's Creative Commons. licence, unless indicated otherwise in a credit line to the material. If material is not included in the article's Creative Commons licence and your intended use is not permitted by statutory regulation or exceeds the permitted use, you will need to obtain permission directly from the copyright holder. To view a copy of this licence, visit http://creativecommons.org/licenses/by/4.0/ The Creative Commons Public Domain Dedication waiver (http://creativecommons.org/publicdomain/zero/1.0/) applies to the data made available in this article, unless otherwise stated in a credit line to the data. 


\section{Background}

Anthocyanins are a chemically diverse class of secondary metabolites belonging to the flavonoid group of plant compound. This versatile group of phenolic molecules, of which more than 635 have been identified to date, are responsible for the different colors (the blues, purples, and reds) of many fruits, seeds, and flowers [1]. Approximately $95 \%$ of all anthocyanins in nature are derived from six major anthocyanidins (aglycones), namely, cyanidin $(\mathrm{Cy})$, pelargonidin $(\mathrm{Pg})$, malvidin $(\mathrm{Mv})$, peonidin $(\mathrm{Pn})$, delphinidin $(\mathrm{Dp})$, and petunidin $(\mathrm{Pt})[2]$. The presence and concentration of these molecules contribute to the pigmentation observed in plant tissues. For example, different blue shades in flowers are attributable to Dp, whereas reddish hues are due to $\mathrm{Cy}$ [3]. In addition to their roles as colorants, certain anthocyanins exert antiviral, antibacterial, and fungicidal activities [4, 5], and accordingly may play roles in protecting plants from infection by pathogenic microorganisms.

Anthocyanins typically show low toxicity to mammals and other vertebrates [6], and numerous studies have indicated that these flavonoids may have health-promoting properties, such as anti-inflammatory, anticarcinogenic, and cardioprotective activity, and efficacy in the control of obesity and alleviation of diabetes. The beneficial effects of anthocyanins, observed in cell line assays, animal studies, and clinical trials, are assumed to be associated with their strong antioxidant capacities [7]. These bioactivities have motivated research on anthocyanins, which are now among the most studied compounds in plant science, and anthocyanin biosynthesis pathways and regulatory mechanisms at the transcriptional level have been thoroughly investigated in many plant species [8, 9]. Although the anthocyanin biosynthesis pathways in maize, snapdragon, and petunia share many common reactions, they also show notable differences, given the large number of genes involved and their multiple complex interactions. On the basis of analyses of the components and content of anthocyanin in mulberry fruit, it has been established that the main component of anthocyanin in mulberry is Cy. However, $\mathrm{Pg}$ and $\mathrm{Dp}$ do not appear to be metabolized normally during mulberry anthocyanin synthesis, the reason for which remains unclear $[10,11]$. These mechanisms ultimately affect the total anthocyanin content in plants, which vary substantially among plant species and even different cultivars of the same species [12].

The mulberry tree (Morus spp.) not only serves as a foodplant for silkworms (Bombyx mori) but has also been traditionally used as a medicinal plant in countries in eastern Asia. In China, mulberry fruit is used as a herbal medicine to protect against liver and kidney damage, improve eyesight, and strengthen the joints, and is also used for its anti-aging effects, and to treat sore throats, fever, hypertension, and anemia [13]. Mulberry fruit contains high amounts of anthocyanins, the differing levels of which are associated with the different colors of fruit [14]. At least 11 anthocyanins have been identified in mulberries, the main components of which are cyanidin-3-O-glucoside and cyanidin-3-O-rutinoside [15.16]. To date, studies on the biosynthesis of mulberry anthocyanins have mainly focused on the characterization and expression of a few selected genes involved in their synthesis [17.18]. However, the available information is limited, and consequently we have yet to gain a complete understanding of anthocyanin biosynthesis in mulberries. Approximately 15 mulberry species are recognized worldwide (M. alba, M. nigra, M. multicaulis, M. atropurpurea, M. bombycis, $M$. mizubo, $M$. wittiorum, M. laevigata, $M$. cathayana, $M$. serrata, $M$. mongolica, M. notabilis, $M$. nigriformis, $M$. yunnanensis, and $M$. australis), among which there are more than 100 mulberry fruit varieties. One of the most prominent differences distinguishing these varieties is the color of the fruit they produce, which can be black, pink, red, or white, and such natural variation among different mulberry types provides valuable opportunities for elucidating the pathways associated with anthocyanin biosynthesis.

In the present study, we focused our efforts in this regard on the two mulberry varieties Da 10 (Morus atropurpurea Roxb.) and Baisang (Morus alba L.) [15]. The leaves of Da10 are heart-shaped, relatively flat, emerald green, smooth, and slightly wrinkled, whereas those of Baisang are heart-shaped, either full or split, flat, dark green, smooth, and without wrinkles. Da 10 is characterized by large purple-black fruit, whereas in contrast, the fruits of Baisang are medium-sized and jade white in color. Previously, Yang et al. comparatively analyzed genetic structures among the 15 species and 4 subspecies of the traditionally classified mulberries. Clustering analysis showed that Nei's genetic consistency coefficient between $M$. atropurpurea and $M$. alba was 0.9575, whereas the genetic distance was 0.0435 , thereby indicating the close relationship between these two species [16].

For the purposes of the present study, we characterized and compared the transcriptional activity of Da 10 and Baisang, at different stages of fruit development defined by the number of days post-anthesis (DPA), namely, S1 (10 DPA), S2 (19 DPA), and S3 (28 DPA), using Illumina sequencing technique. The identities of the differentially expressed genes (DEGs) were determined based on reference to the Gene Ontology (GO) and Kyoto Encyclopedia of Genes and Genomes (KEGG) databases. Transcriptome data were validated by comparing RNA-Seq results with the findings of quantitative real-time PCR (qPCR). We also investigated the regulatory role of transcription factors in anthocyanin 
synthesis to gain a better understanding of the mechanisms underlying fruit color development in mulberry.

\section{Results}

\section{Classes of color compounds in mulberry fruit}

To examine the biochemical factors underlying the lack of color development in white mulberry fruit, we compared the anthocyanin content of the two mulberry varieties $\mathrm{Da} 10$ and Baisang at different stages of fruit development. In Da 10, fruit color changed from green, to purple, and subsequently to black at stages S1, S2, and S3, respectively (Fig. 1). For the Baisang variety, mulberries changed from green, to light green, and then to white at stages S1, S2, and S3, respectively (Fig. 1). The anthocyanin content in $\mathrm{Da} 10$ comprised $\mathrm{Cy}$ and Pg. At S1, neither of these two anthocyanins were detected, whereas at stage 2, Cy and Pg levels were $23.533 \mu \mathrm{g} / \mathrm{g}$ and $4.197 \mu \mathrm{g} / \mathrm{g}$, respectively. The levels of $\mathrm{Cy}$ and $\mathrm{Pg}$ increased during the ripening of $\mathrm{Da} 10$, reaching 375.29 and $24.423 \mu \mathrm{g} / \mathrm{g}$, respectively, at stage S3. In Baisang, Cy was the only anthocyanin detected, the levels of which ranged from $4.87 \mu \mathrm{g} / \mathrm{g}$ at $\mathrm{S} 2$ to $10.957 \mu \mathrm{g} / \mathrm{g}$ at $\mathrm{S} 3$ (Table 1). Thus, although the fruit of Baisang appear white, they contain low levels of Cy. None of the other anthocyanins $\mathrm{Dp}, \mathrm{Pn}$, and $\mathrm{Mv}$ were detected at any developmental stage in either of the two varieties.

\section{Transcriptome sequencing, clustering, and functional enrichment of DEGs}

As a consequence of quality filtering, we obtained 33.77-74.56 million high-quality 150-bp paired-end reads. Having removed rRNA reads, the sequencing reads were mapped to the Morus notabilis Schneid. reference genome (https://morus.swu.edu.cn/morusdb/), resulting in the annotation of more than 27,085 genes, including 1735 new genes (Table S1).

DEGs were identified using the edgeR package (http:// www.rproject.org/). Comparing the sequenced transcripts obtained at stages S1 and S2, we detected 5513 and 3973 DEGs in the fruit of Da 10 and Baisang, respectively, whereas our comparison between stages S2 and S3 enabled us to identify 7204 and 5359 in the fruits of Da 10 and Baisang, respectively. On the basis of the comparison between the two varieties, we detected 4256, 5612, and 5226 DEGs at developmental stages S1, S2, and $\mathrm{S} 3$, respectively (Fig. 2a).

To analyze DEG expression patterns, the expression data at different stage were normalized to $0, \log _{2}(\mathrm{v} 1 / \mathrm{v} 0)$, and $\log _{2}(\mathrm{v} 2 / \mathrm{v} 0)$, and then clustered using Short Timeseries Expression Miner software (STEM). DEGs in the fruit of the two varieties were clustered into eight profiles based on gene expression patterns. DEGs in profiles 0,1 , and 3 were significantly enriched in Da 10 , whereas those in profiles 0,3 , and 7 were significantly enriched in Baisang (Fig. 2b). The DEGs in profiles 0 and 3 were downregulated in both genotypes. To examine the relationship between the enriched genes and metabolite accumulation, the DEGs in profiles 0,3 , and 7 were annotated for both varieties. Profile 7 contained 25 and 10 DEGs in Da 10 and Baisang, respectively, that are related to the biosynthesis of secondary metabolites. In profile 7 , a larger number of the genes involved in carbohydrate, lipid, and energy metabolism were from Baisang (Fig. 2c). Pathway enrichment analysis showed
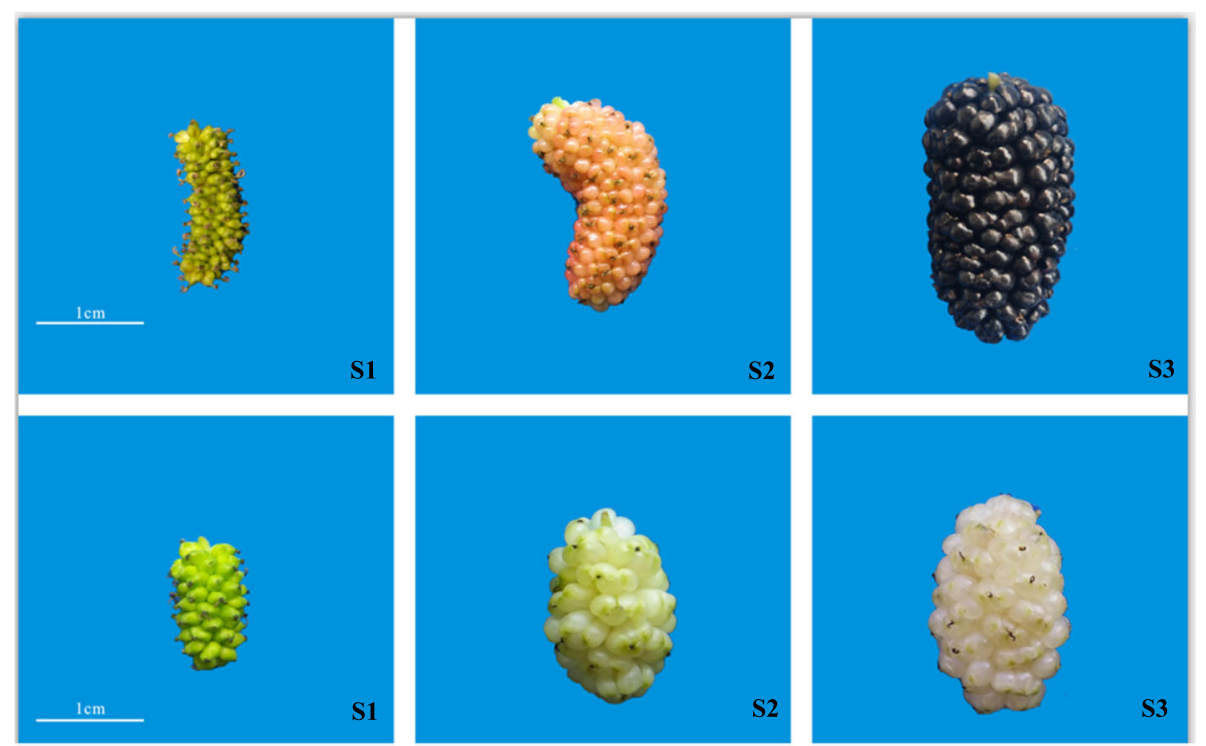

Fig. 1 Representative images of mulberries from the two varieties Da 10 (upper row) and Baisang (lower row) at different developmental stages, S1 (10 days post-anthesis, DPA), S2 (19 DPA), and S3 (28 DPA) 
Table 1 Anthocyanin content $(\mu \mathrm{g} / \mathrm{g}$ ) in mulberries from the two varieties, Da 10 (black) and Baisang (white), at different developmental stages, S1 (10 days post-anthesis, DPA), S2 (19 DPA), and S3 (28 DPA)

\begin{tabular}{llllll}
\hline Sample & Delphinidin & Cyanidin & Pelargonidin & Peonidin & Malvidin \\
\hline Da 10-S1 & 0.00 & 0.00 & 0.00 & 0.00 & 0.00 \\
Da 10-S2 & 0.00 & $23.533 \pm 3.371$ & $4.197 \pm 0.566$ & 0.00 & 0.00 \\
Da 10-S3 & 0.00 & $375.29 \pm 2.851$ & $24.423 \pm 1.188$ & 0.00 & 0.00 \\
Baisang-S1 & 0.00 & 0.00 & 0.00 & 0.00 & 0.00 \\
Baisang-S2 & 0.00 & $4.87 \pm 0.896$ & 0.00 & 0.00 & 0.00 \\
Baisang-S3 & 0.00 & $10.957 \pm 1.381$ & 0.00 & 0.00 & 0.00 \\
\hline
\end{tabular}

that tyrosine, phenylalanine, tryptophan, phenylpropanoid, flavonoid, and anthocyanin biosynthesis were significantly enriched in profile 7 for $\mathrm{Da} 10$, which indicates that anthocyanins were synthesized from the precursor phenylalanine, via the biosynthesis of phenylpropanoid, flavonoid, and anthocyanin. In Baisang, profile 7 contained genes involved in fructose and mannose metabolism, fatty acid metabolism, glycolysis/gluconeogenesis, and fatty acid biosynthesis (Fig. 2d).

The anthocyanin biosynthesis pathway in mulberry fruit Based on the data of transcriptome sequencing and DEG expression patterns to identify the key transcripts involved in anthocyanin metabolism, we compared the abundance of genes in the Da 10 and Baisang transcriptomes. We accordingly found that three genes involved in tyrosine, phenylalanine, and tryptophan biosynthesis, chorismate mutase (CM), arogenate dehydratase (PDT), and aspartate-prephenate aminotransferase (PAT), were upregulated in Da 10 but not in Baisang (Table S2). Other genes overexpressed in Da 10 relative to Baisang included those related to phenylpropanoid, flavonoid, and anthocyanin biosynthesis (Table S2, Fig. S1). We thus deduced that pathways involved in phenylalanine, tyrosine, tryptophan, phenylpropanoid, flavonoid, and anthocyanin biosynthesis are essential for the development of the $\mathrm{Da} 10$ phenotype. Targeted metabolomics data indicated similar trends in the metabolites associated with these pathways.

To obtain a global picture of the anthocyanin biosynthesis pathway in mulberry fruit, we compared the transcript levels of genes involved in anthocyanin synthesis, and the main related metabolic branches, in Da 10 and Baisang. Figure 3 presents a schematic representation of anthocyanin metabolism with its core metabolites and enzymes in mulberry fruit, highlighting the key steps

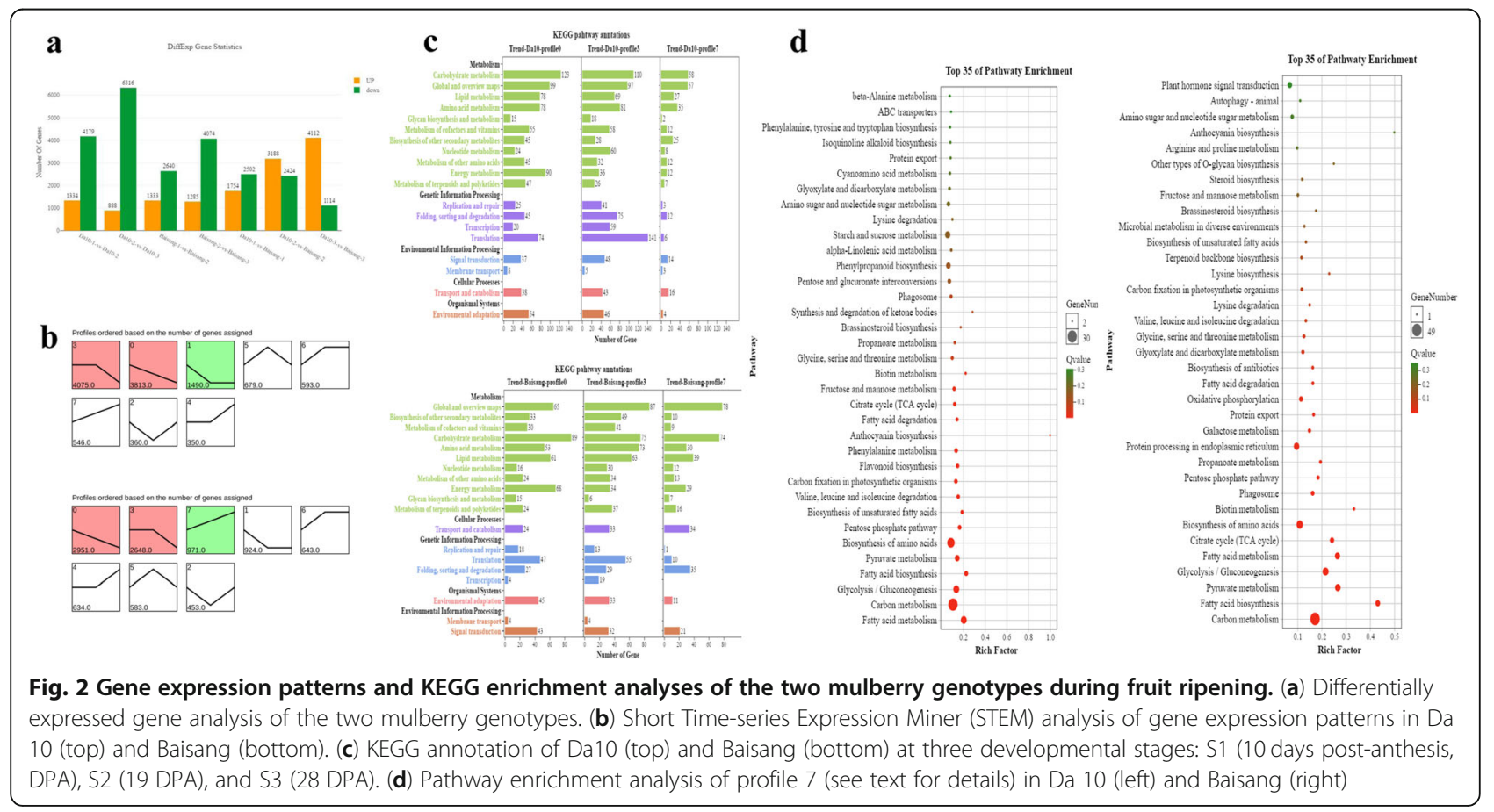




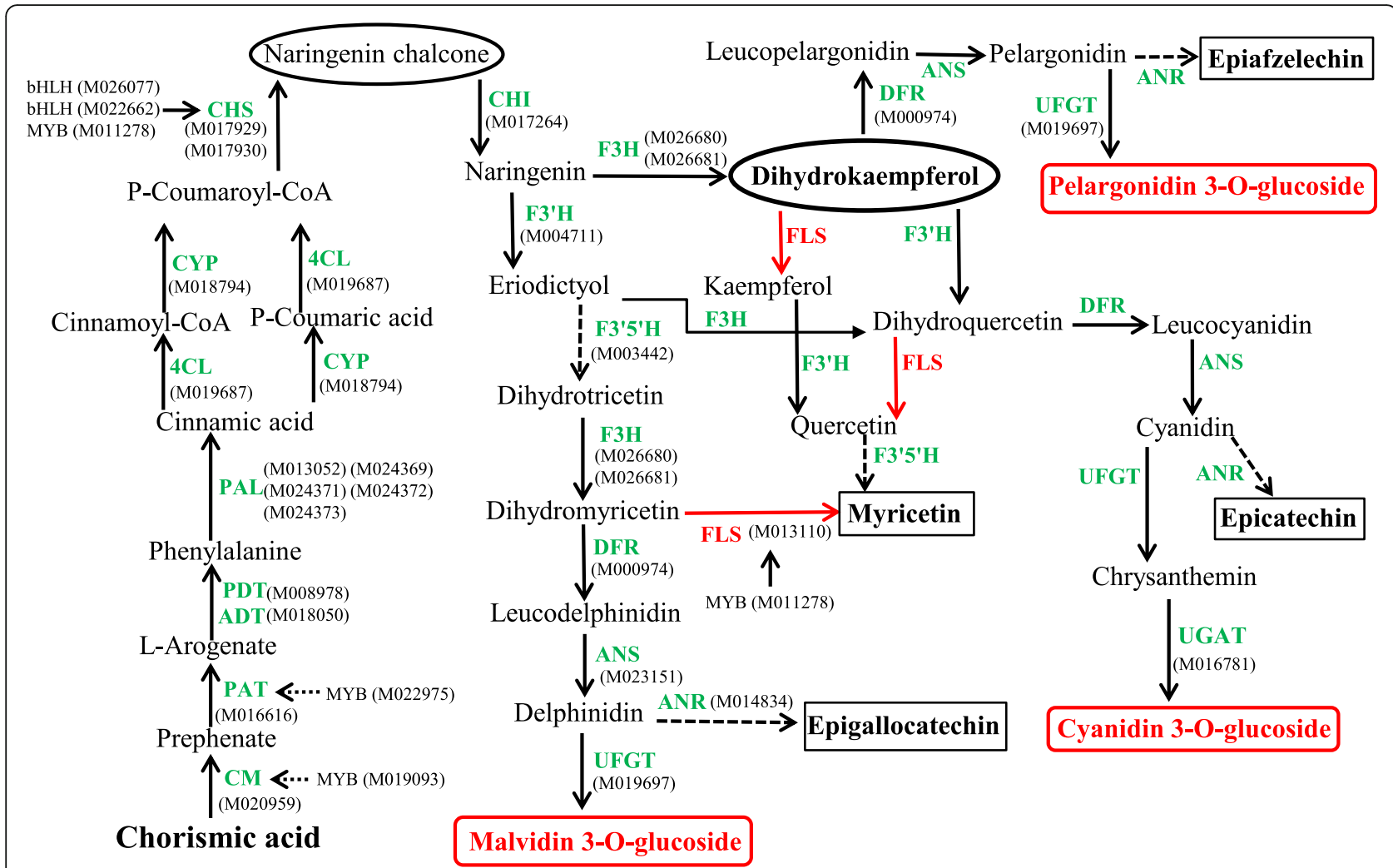

Fig. 3 A schematic representation of the anthocyanin biosynthesis pathway in mulberry fruit. Genes colored green are involved in all anthocyanin biosynthesis pathways, whereas those colored red control myricetin synthesis

that differ between the two mulberry varieties. Chalcone synthase (CHS) expression levels were found to be more than 40 times higher in black mulberries than in white fruit. A further gene upregulated in Da 10 is naringenin [2-oxoglutarate 3-dioxygenase (F3H, M026681)], the expression levels of which in black mulberries were found to be approximately 12 times higher than those in white fruit (Fig. 4 and Fig. S2). Expression at this level is expected to produce large amounts of another intermediate metabolite, dihydrokaempferol. The upregulation of these two pathways would enhance the supply of naringenin chalcone and dihydrokaempferol, thereby promoting the synthesis of anthocyanins in black mulberries (Fig. 3).

At the early stage of fruit development, both black and white mulberries showed similar levels of anthocyanins and patterns of gene expression. The presence of both $\mathrm{Cy}$ and Pg in black mulberries during the latter stages of development indicates that the anthocyanin biosynthesis pathway is probably induced at a point relatively far downstream of the late reactions catalyzed by enzymes such as dihydroflavonol 4-reductase (DFR), anthocyanidin synthase (ANS), or anthocyanidin 3-O-glucosyltransferase (UFGT). In contrast, the presence of myricetin in the white fruit indicates that the expression of flavonol synthase/flavanone 3-hydroxylase (FLS) was significantly upregulated (Figs. 3 and 4).

\section{Quantitation of related metabolites in the anthocyanin synthesis of mulberry fruit}

On the basis of the annotated anthocyanin synthesis pathway in mulberry fruit, we performed an absolute quantitative analysis of metabolites, namely, 10 flavonoids and their precursors, including l-phenylalanine, naringenin, and cyanidin 3-O-glucoside, via the detection of targeted metabolites (Table 2). With respect to the phenylpropanoid biosynthesis pathway, we found that in Baisang, the content of l-phenylalanine and hydroxy-methoxycinnamate at the three stages of fruit development were higher than those in Da 10. Similarly, in the flavonoid biosynthesis pathway, the content of naringenin, dihydrokaempferol, eriodictyol, and dihydroquercetin at stages S1 and S2 in Baisang were higher than those in Da 10, whereas at stage 3, the content of these compounds was lower in Baisang than in Da 10. The content of dihydromyricetin at stage S3 was also found to be higher in Da 10 than in Baisang, although was not detected in either genotypes at stages S1 and S2. In the anthocyanin biosynthesis pathway of Baisang, we observed no significant changes in the content of 


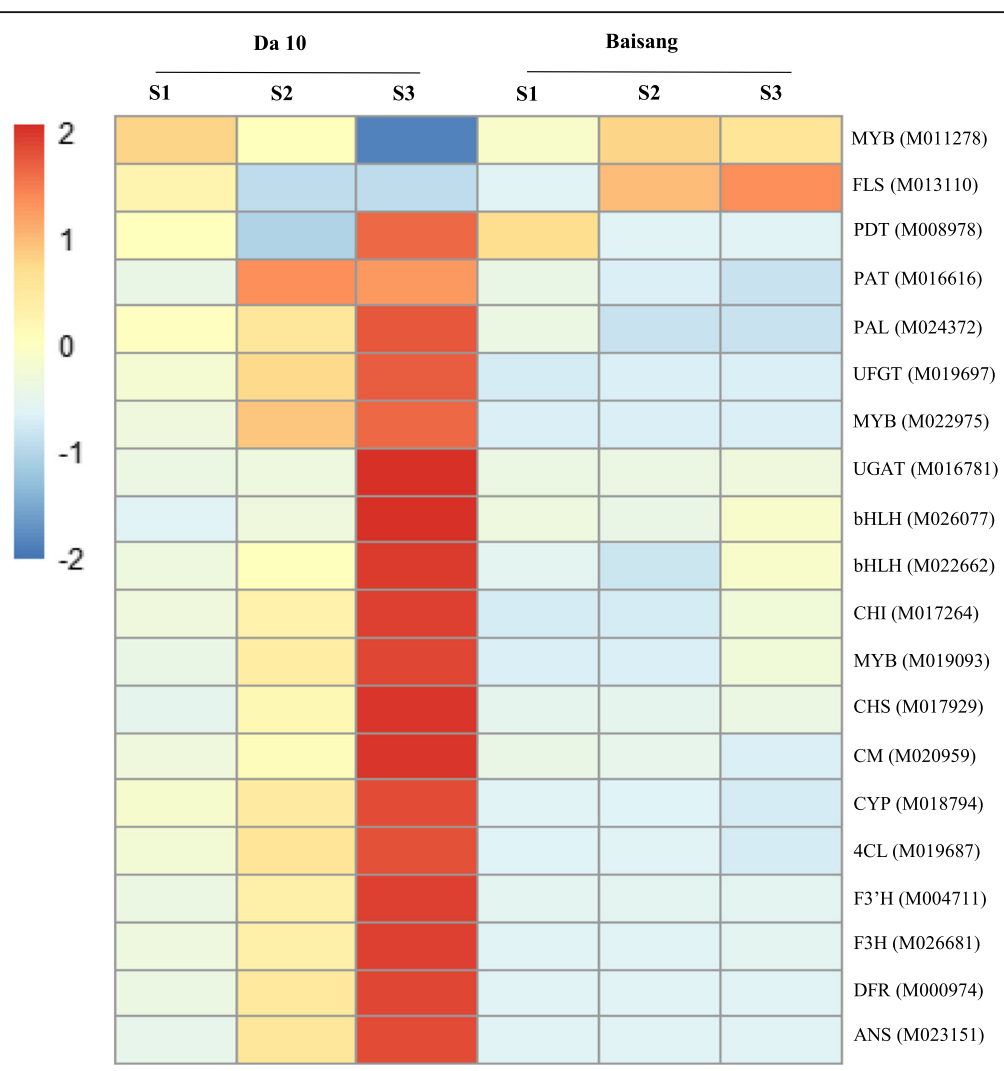

Fig. 4 Expression levels of the 15 core genes and five transcription factors involved in anthocyanin biosynthesis in mulberry fruit. Each square represents the transcription level in the two mulberry varieties (Da 10 and Baisang) at different developmental stages, S1 (10 days postanthesis, DPA), S2 (19 DPA), and S3 (28 DPA). Different color indicates differences in the level of gene expression, from high (red) to low (blue)

cyanidin 3-O-glucoside or cyanidin 3-O-rutinoside in the three developmental stages, with only low level $(25.10 \mu \mathrm{g} / \mathrm{g}$ and $13.01 \mu \mathrm{g} / \mathrm{g}$, respectively) being detected at stage 3. Contrastingly, these two metabolites gradually increased in Da 10 concomitant with the development of fruit, reaching maximum levels of $9417.323 \mu \mathrm{g} / \mathrm{g}$ and $3227.725 \mu \mathrm{g} / \mathrm{g}$, respectively, at stage 3 . In the case of Baisang, whereas l-phenylalanine and other precursors were abundant, the amounts of naringenin and other intermediate products were considerably lower than those detected in Da 10 during fruit development.

\section{Genes related to anthocyanin biosynthesis}

The early genes include CM, arogenate dehydratase/prephenate dehydratase 6 (PDA), 4-coumarate-CoA ligase (4CL), phenylalanine ammonia-lyase (PAL), CHS, chalcone-flavonone isomerase $(\mathrm{CHI})$, and $\mathrm{F} 3{ }^{\prime} \mathrm{H}$, which contribute to the formation of dihydroflavonols. The late genes, DFR, ANS, UFGT, and cyanidin-3-O-glucoside 2$\mathrm{O}$-glucuronosyltransferase (UGAT), play roles in the generation of anthocyanins (Table S2, Fig. 3). In the present study, we identified 15 core genes and five transcription factors related to anthocyanin biosynthesis (Table S2), among which, 18 were upregulated. Only
FLS and the MYB transcription factor (M011278) were downregulated in black mulberries relative to white fruit (Fig. 4). Notably, both early (CM, PAT, PAL, CYP, 4CL, and CHS) and late (DFR, ANS, UFGT, and UGAT) genes were significantly downregulated in white mulberries relative to black mulberries (Fig. 4). The outcome of the downregulation of UFGT would be a reduction in the synthesis of $\mathrm{Mv}$ and $\mathrm{Pg}$, whereas downregulation of UGAT would lead to lower levels of $\mathrm{Cy}$.

The formation of flavonols from dihydroflavonols is catalyzed by FLS, which acts on dihydrokaempferol, dihydroquercetin, and dihydromyricetin to produce kaempferol, quercetin, and myricetin, respectively. Compared with the black mulberry, we found that FLS expression was upregulated in the white mulberry (Fig. 4). It is conceivable that in the latter variety, the low levels of anthocyanin intermediates contribute to the downregulation of the core anthocyanin biosynthesis pathway genes, thereby inhibiting the synthesis of cyanidin, whereas the upregulation of FLS gene expression would block the synthesis of anthocyanins.

Our analysis of the mulberry transcriptome data resulted in the annotation of 1217 transcription factors, among which, 899 were found to be differentially 


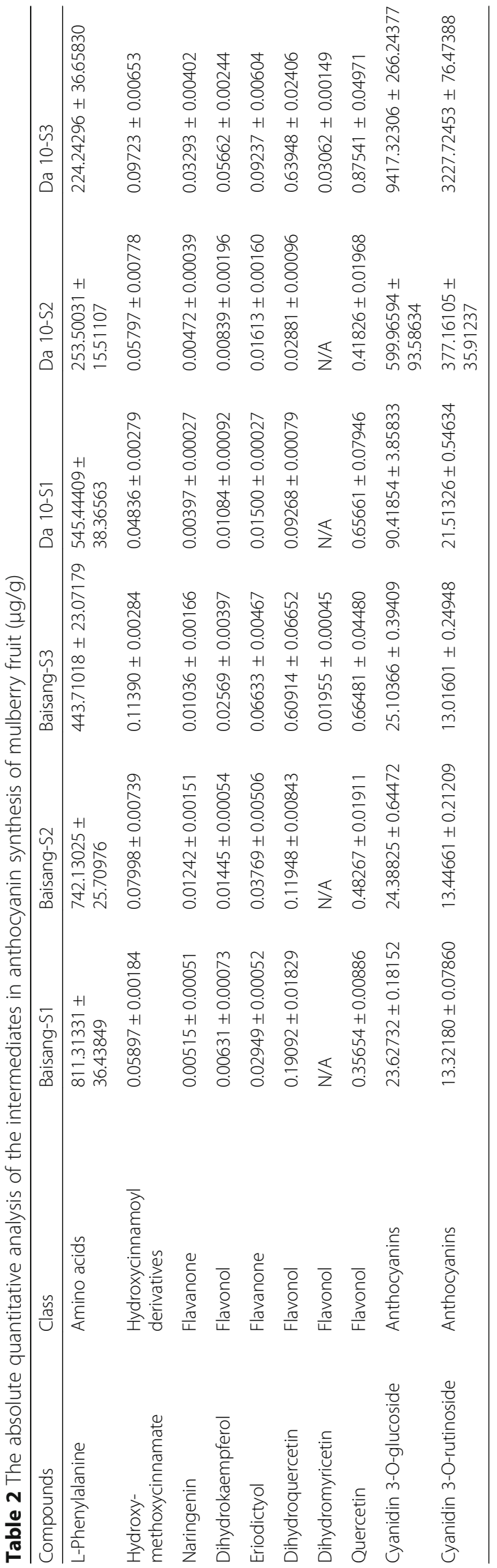


expressed, including 78 MYB transcription factors, 83 bHLH (basic helix-loop-helix) transcription factors, and 31 MYB-related transcription factors. Most of the transcription factors involved in the regulation of genes related to the anthocyanin biosynthesis pathway belong to the MYB and bHLH families. In the present study, we found that the expression of transcription factors differed markedly between Da 10 and Baisang. Those in the MYB (M022975 and M019093) and bHLH (M026077 and M022662) families involved in anthocyanin biosynthesis were upregulated in black mulberries relative to white mulberries, whereas MYB (M011278) showed an inconsistent expression pattern.

\section{Validation of the expression of core genes and transcription factors using qPCR}

In order to verify the expression levels of core genes and transcription factors related to mulberry anthocyanin synthesis, we selected the Morus010170 gene as a reference gene, based on its stable expression in all stages of fruit development in both genotypes. We then validated the expression of 19 genes using qPCR (Table 3, Fig. S2). The expression levels of these 19 genes at different developmental stages were consistent with those determined using RNA-Seq. All 19 genes (particularly CHS, F3' $\mathrm{H}, \mathrm{F} 3 \mathrm{H}, \mathrm{DFR}$, UFGT, and ANS) were found to be expressed at considerably higher levels in Da 10 than in
Baisang. Moreover, in all samples, gene expression was significantly higher at stages S2 and S3 than at stage S1. These results accordingly indicate that these 19 genes are involved in the biosynthesis of $\mathrm{Cy}$ and $\mathrm{Pg}$ via the mulberry anthocyanin biosynthesis pathway. Furthermore, we speculate that the downregulated expression of related genes (DFR, ANS, UFGT, and UGAT) and the upregulated expression of FLS may contribute to the suppression of anthocyanin synthesis in Baisang fruit.

\section{Discussion \\ Anthocyanin compounds and transcriptome analysis of mulberry fruit}

We investigated the biosynthesis of anthocyanins in mulberry by comparison of their levels in the mulberry varieties Da 10 (black fruit) and Baisang (white fruit). The anthocyanin content in $\mathrm{Da} 10$ comprised $\mathrm{Cy}$ and $\mathrm{Pg}$, whereas Cy was the only anthocyanin detected with the low level in Baisang. The presence in mulberries of the two anthocyanins $\mathrm{Cy}$ and $\mathrm{Pg}$, both of which are cyanidin derivatives, is consistent with the findings of earlier studies $[17,18]$. These results confirm, at the metabolite level, the genotype-dependent difference in the accumulation of anthocyanins in mulberry varieties, as well as the association between fruit color and anthocyanin levels. In this regard, $\mathrm{Cy}$ and Pg appear to be the main anthocyanins determining the color of mulberry fruit.

Table 3 Expression levels of core genes and transcription factors involved in anthocyanin biosynthesis in mulberry fruit measured by $\mathrm{qPCR}$

\begin{tabular}{|c|c|c|c|c|c|c|}
\hline Gene_ID & Da 10-S1 & Da 10-S2 & Da 10-\$3 & Baisang-S1 & Baisang-\$2 & Baisang-\$3 \\
\hline Morus020959 & $1.18222 \pm 0.15889^{b c}$ & $1.88258 \pm 0.25197^{b}$ & $7.77758 \pm 1.14978^{\mathrm{a}}$ & $0.72409 \pm 0.03334^{\mathrm{cd}}$ & $0.62440 \pm 0.00670^{c d}$ & $0.18361 \pm 0.01232^{d}$ \\
\hline Morus016616 & $1.06168 \pm 0.06172^{d}$ & $2.73895 \pm 0.04598^{b}$ & $2.96170 \pm 0.12892^{\mathrm{a}}$ & $1.35136 \pm 0.14360^{c}$ & $0.74855 \pm 0.00177^{e}$ & $0.51202 \pm 0.02061^{f}$ \\
\hline Morus008978 & $2.08985 \pm 1.04272^{b}$ & $0.55526 \pm 0.03775^{c}$ & $3.98063 \pm 0.54728^{\mathrm{a}}$ & $4.43413 \pm 1.67624^{a}$ & $1.39548 \pm 1.28161^{b c}$ & $1.24409 \pm 0.13577^{\mathrm{bc}}$ \\
\hline Morus024372 & $1.16249 \pm 0.18139^{c}$ & $2.91039 \pm 0.27492^{b}$ & $8.06253 \pm 0.69500^{\mathrm{a}}$ & $0.21913 \pm 0.02372^{d}$ & $0.21216 \pm 0.02373^{d}$ & $1.03741 \pm 0.02173^{c}$ \\
\hline Morus019687 & $1.22060 \pm 0.20707^{c}$ & $3.37482 \pm 0.08522^{b}$ & $6.79941 \pm 0.43346^{\mathrm{a}}$ & $0.36948 \pm 0.04739^{d}$ & $0.34017 \pm 0.02607^{d}$ & $0.10043 \pm 0.00688^{d}$ \\
\hline Morus018794 & $1.12094 \pm 0.11790^{c}$ & $2.51933 \pm 0.08317^{b}$ & $5.15479 \pm 0.27124^{\mathrm{a}}$ & $0.33514 \pm 0.01251^{d}$ & $0.19838 \pm 0.01246^{d}$ & $0.03483 \pm 0.00088^{d}$ \\
\hline Morus017929 & $1.25044 \pm 0.23356^{c}$ & $60.05397 \pm 6.67163^{b}$ & $204.42567 \pm 16.72710^{a}$ & $0.24663 \pm 0.05032^{c}$ & $0.70137 \pm 0.11956^{c}$ & $9.38162 \pm 1.11148^{c}$ \\
\hline Morus017264 & $1.16249 \pm 0.18139^{c}$ & $2.91039 \pm 0.27492^{b}$ & $8.06253 \pm 0.69500^{a}$ & $0.21913 \pm 0.02372^{d}$ & $0.21216 \pm 0.02373^{d}$ & $1.03741 \pm 0.02173^{c}$ \\
\hline Morus004711 & $1.11432 \pm 0.14113^{c}$ & $9.52750 \pm 1.83368^{b}$ & $19.26487 \pm 0.73340^{a}$ & $0.01153 \pm 0.00292^{c}$ & $0.03839 \pm 0.00177^{c}$ & $0.16674 \pm 0.02614^{c}$ \\
\hline Morus026681 & $1.18738 \pm 0.19339^{c}$ & $4.39685 \pm 0.33282^{b}$ & $12.12864 \pm 1.21909^{a}$ & $0.12576 \pm 0.00747^{d}$ & $0.17875 \pm 0.00741^{d}$ & $0.38565 \pm 0.01410^{\mathrm{cd}}$ \\
\hline Morus000974 & $1.16340 \pm 0.23216^{c}$ & $6.59875 \pm 0.63817^{b}$ & $17.33984 \pm 1.65727^{\mathrm{a}}$ & $0.00354 \pm 0.00181^{c}$ & $0.01215 \pm 0.00202^{c}$ & $0.00158 \pm 0.00047^{c}$ \\
\hline Morus023151 & $1.16012 \pm 0.17505^{c}$ & $11.52419 \pm 0.24537^{b}$ & $27.29133 \pm 1.82231^{a}$ & $0.00568 \pm 0.00231^{c}$ & $0.01615 \pm 0.00323^{c}$ & $0.00420 \pm 0.00125^{c}$ \\
\hline Morus013110 & $1.17889 \pm 0.16729^{b c}$ & $0.20182 \pm 0.01650^{d}$ & $0.44767 \pm 0.15683^{d}$ & $0.94758 \pm 0.04242^{c}$ & $1.97049 \pm 0.17624^{\mathrm{ab}}$ & $2.02151 \pm 0.17312^{\mathrm{a}}$ \\
\hline Morus019697 & $1.04230 \pm 0.08904^{c}$ & $3.33870 \pm 0.05326^{\mathrm{b}}$ & $6.15405 \pm 0.27407^{\mathrm{a}}$ & $0.00067 \pm 0.04000^{d}$ & $0.00039 \pm 0.04000^{d}$ & $0.00024 \pm 0.00007^{d}$ \\
\hline Morus022662 & $0.98671 \pm 0.01461^{c d}$ & $1.18920 \pm 0.14632^{\mathrm{bd}}$ & $3.86468 \pm 0.45449^{a}$ & $1.15804 \pm 0.06608^{b}$ & $0.67690 \pm 0.00231^{c}$ & $1.37798 \pm 0.04622^{b}$ \\
\hline Morus026077 & $0.94035 \pm 0.05657^{c}$ & $1.69939 \pm 0.32301^{b}$ & $8.28561 \pm 1.13372^{\mathrm{a}}$ & $2.35982 \pm 0.52593^{b}$ & $0.77495 \pm 0.12562^{c}$ & $2.18711 \pm 0.25806^{b}$ \\
\hline Morus019093 & $1.15571 \pm 0.15154^{b}$ & $5.54870 \pm 0.14925^{b}$ & $14.94315 \pm 0.25314^{\mathrm{a}}$ & $0.04933 \pm 0.01419^{d}$ & $0.07291 \pm 0.00429^{d}$ & $1.44544 \pm 0.27548^{c}$ \\
\hline Morus022975 & $1.43737 \pm 0.38951^{c}$ & $5.83052 \pm 0.18116^{b}$ & $8.98590 \pm 1.54734^{a}$ & $0.14698 \pm 0.00992^{d}$ & $0.03833 \pm 0.00339^{d}$ & $0.01377 \pm 0.00210^{d}$ \\
\hline Morus011278 & $0.96787 \pm 0.02825^{d}$ & $0.81453 \pm 0.09266^{e}$ & $0.34772 \pm 0.00691^{f}$ & $2.83443 \pm 0.08113^{\mathrm{a}}$ & $2.47529 \pm 0.12662^{b}$ & $1.97841 \pm 0.11099^{c}$ \\
\hline
\end{tabular}

Note: In the same row values with different small letter superscripts mean significant difference $(P<0.05)$. 
Once formed, the unstable Cy would be converted to the colorless epicatechin, which would permanently prevent the formation of stable color pigments via later glycosylation and other reactions.

Based on a comparative transcriptome analysis, we annotated more than 27,085 genes, including 1735 new genes. Genes that were differentially expressed between Da 10 and Baisang were detected 4256, 5612, and 5226 DEGs at developmental stages S1, S2, and S3, respectively (Fig. 2a).

\section{The anthocyanin biosynthesis pathway in mulberry fruit} Anthocyanin synthesis in the leaves, fruits, and flowers of Arabidopsis thaliana, grape, and hyacinth has been shown to be associated with three secondary metabolic pathways, namely, the phenylpropanoid, flavonoid, and anthocyanin biosynthesis pathways [19-21]. The lack of color development in white mulberries would require a complete blockage of the anthocyanin biosynthesis pathway, which presumably occurs prior to the formation of Dp and Cy [22]. In this study, three genes involved in tyrosine, phenylalanine, and tryptophan biosynthesis, CM, PDT, and PAT, were upregulated in Da 10 but not in Baisang (Table S2). CHS and F3H expression levels were found to be more than 40 times and 12 times higher respectively, in black mulberries than in white fruit (Fig. 4 and Fig. S2). It is assumed that such overexpression would lead to a large production of the intermediate metabolite naringenin chalcone in black mulberry. CHS catalyzes the first reaction of anthocyanin biosynthesis, and subsequently contributes to the formation of chalcone (intermediate), which is the primary precursor of flavonoids [23]. Consequently, when chalcone synthesis is constrained, both anthocyanin production and that of nearly all other flavonoids, is affected [24]. DFR, ANS, UFGT and FLS hydroxylation reactions during anthocyanin biosynthesis pathway are essential to the formation of different types of anthocyanins, and eventually produce different colors. Indeed, anthocyanin accumulation is associated with the expression of the genes encoding the enzymes that participate in these reactions [25-27].

Quantitation of related metabolites in the anthocyanin synthesis of mulberry fruit showed that the content of cyanidin 3-O-glucoside and cyanidin 3-O-rutinoside in Baisang were substantially lower than those in Da 10. In both genotypes, quercetin accumulates at stage S1; however, whereas it gradually increased in Baisang during the latter stages of fruit development, it was found to decrease in Da 10, thereby indicating that the upregulated expression of the FLS gene promotes the conversion of dihydroquercetin to quercetin, and further inhibits anthocyanin synthesis.

\section{Expression of core genes and transcription factors in mulberry fruit}

Genes that regulate the anthocyanin biosynthesis pathway are generally classified as early and late genes [28]. In this study, both early (CM, PAT, PAL, CYP, 4CL, and $\mathrm{CHS}$ ) and late (DFR, ANS, UFGT, and UGAT) genes were significantly downregulated in white mulberries relative to black mulberries. And, FLS expression was upregulated in the white mulberry (Fig. 4). Chalcone synthase is a plant-specific polyketide synthase that plays a key role in flavonoid biosynthesis. In strawberries, CHS is expressed not only in the petals but also in the fruit (in which its transcripts are abundant) [29] during strawberry ripening, and upregulation of the CHS, F3' $\mathrm{H}$, DFR, and UFGT genes corresponds to an increase in fruit enzymatic activity [30], resulting in the accumulation of anthocyanin at the ripe red stage. Similar observations have been made with respect to ANS expression and anthocyanin accumulation in Allium cepa [31], Duchesnea indica [32], and M. alba [17, 33].

The functions of MYB transcription factors in the regulation of genes associated with anthocyanin accumulation have been reported in several plant species, including rice (Oryza sativa) [34], A. thaliana [35], maize (Zea mays) [36], petunia (Petunia hybrida) [37], grapevine (Vitis vinifera L.) [38], apple (Malus domestica) [39], and poplar (Populus tremuloides) [40], based on genetic and molecular analyses. The bHLH proteins form the second largest family of transcription factors in plants, in which they play an important role of metabolic, physiological, and developmental processes [41]. The present results showed that the expression of transcription factors differed markedly between Da 10 and Baisang. MYB (M022975 and M019093) and bHLH (M026077 and M022662) were upregulated in black mulberries relative to white mulberries, whereas MYB (M011278) showed an inconsistent expression pattern.

\section{Conclusion}

The biochemical and molecular findings of this study provide a more comprehensive insight into those mechanisms underlying the synthesis and accumulation of anthocyanins in different mulberry genotypes. We observed substantial increases in levels of the two anthocyanins $\mathrm{Cy}$ and Pg during late ripening of $\mathrm{Da} 10$ fruits, whereas in Baisang, only Cy was detected, although only at low levels and exclusively during the late stage of fruit development. The high content of anthocyanins in black mulberries was associated with an upregulation of genes involved in the initial steps of anthocyanin synthesis (CM, CHS, and $\mathrm{CHI}$ ), which produce the intermediates, chalcone and dihydroflavonol. The expression of genes that ultimately yield $\mathrm{Cy}, \mathrm{Pg}$, and $\mathrm{Mv}$ during the latter steps of anthocyanin synthesis (DFR, ANS, UFGT, and 
UGAT) was also upregulated in black mulberries. In contrast, the low anthocyanin content in white mulberries can be attributed primarily to the downregulation of early genes (MYB, bHLH, CHS, and CHI), and an upregulated expression of FLS, leading to the generation of quercetin and/or myricetin.

\section{Methods}

\section{Plants and reagents}

Fruit samples from the two mulberry genotypes [black fruit (Da 10 variety) and white fruit (Baisang variety)] (Fig. 1) were collected from the mulberry garden of the Sericultural Research Institute, Sichuan Academy of Agricultural Sciences, China. Nine plants of each variety were randomly selected, and 20 fruits from each plant were collected at each of the three assessed developmental stages, which were defined as follows: S1 (green, 10 DPA), S2 (semi-mature, 19 DPA), and S3 (mature, 28 DPA). Fruits harvested at the same stage in the same variety were mixed and divided into three replicate groups, and then frozen in liquid nitrogen and immediately stored at $-80^{\circ} \mathrm{C}$ for subsequent anthocyanin determination and transcriptome analysis.

Anthocyanin analytical standards (for cyanidin, delphinidin, peonidin, pelargonidin, and malvidin) were purchased from Sigma-Aldrich (St. Louis, MO, USA). Iodomethane, dimethyl carbonate, dimethyl phosphate, and acetonitrile were provided by Sangon Biotech (Shanghai, China). Other reagents used were of analytical grade.

\section{Determination of mulberry anthocyanin levels}

The extraction and determination of anthocyanins from mulberry fruit were performed using a previously described method [42]. Anthocyanin extraction was carried out using acidified methanol (methanol and $1.0 \mathrm{~N} \mathrm{HCl}$, $85: 15, \mathrm{v} / \mathrm{v})$, assisted by ultrasonic disruption. The extracts were concentrated by evaporation at $50^{\circ} \mathrm{C}$ using a rotary evaporator, and were then re-dissolved in acidified methanol. Individual anthocyanins were separated and quantified using a high-performance liquid chromatography (HPLC) system (1260 Infinity II LC, Agilent Technologies Inc., CA, USA), coupled to a dual wavelength ultraviolet-visible detector and a data acquisition system (Millennium Chromatography Manager version 2.15.01; Waters Corporation, MA, USA). A reversed-phase chromatography column (Supelcosil LC-18-dB, $25 \mathrm{~cm} \times 4.6$ $\mathrm{mm}$ i.d.; Agilent) was used at room temperature.

\section{RNA extraction and sequencing}

Total RNA isolation was performed using an RN09EASY spin plus Plant Kit (Aidlab Biotech, Beijing, China) based on a previously described method [43]. The integrity of the extracted RNA was verified by agarose gel electrophoresis, and the concentration was measured using a 2100 Bioanalyzer (Agilent Technologies, CA, USA). cDNA libraries were constructed for each sample using enriched mRNA. High-quality RNA was used for subsequent RNA sequencing, which was performed using the HiSeq 2500 platform (Illumina, San Diego, CA, USA) at Guangzhou Gene Denovo Biotechnology Co. Ltd. (Guangzhou, China).

\section{Normalization and identification of DEGs}

Sequencing reads were mapped to the reference sequences of $M$. notabilis using SOAPaligner/SOAP2 [44]. Gene expression levels were measured as fragments per kilobase of exon model per million reads mapped (FPKM), with the longest transcript being used to calculate the FPKM.

DEGs were identified by comparing expression levels of the transcripts of Da 10 and Baisang at the same developmental stage. For the correction of multiple testing, the false discovery rate (FDR) was analyzed for adjustment of the p-value threshold [45]. To assess changes in gene expression patterns during fruit ripening within the two genotypes, expression pattern analysis was performed using Short Time-series Expression Miner (STEM, version 1.3.8) [46] based on all the DEGs of Da 10 and Baisang mulberries.

\section{Annotation and GO and KEGG functional classification}

All expressed genes were functionally annotated using the following databases: the NCBI nonredundant protein database $(\mathrm{Nr})$, the Kyoto Encyclopedia of Genes and Genomes (KEGG), the Clusters of Orthologous Groups of proteins database (COG), and the Swiss-Prot database. For the purposes of annotation, we used BLASTX with an e-value cutoff of $1 \mathrm{e}-5$ in Blast2GO [43]. For transcript sequences, the matched protein with the highest similarity rate was determined to be the optimal annotation.

Gene Ontology (GO) classification was performed for the upregulated genes in Da 10 and Baisang using Web Gene Ontology Annotation Plot (WEGO) [43], and a $\chi^{2}$ test was used to identify GO terms over-represented (or under-represented) in one variety compared with the other. To identify KEGG pathways, the numbers of upand downregulated genes in each genotype were compared with those in the reference set.

\section{Metabolite validation and expression analysis}

To analyze the accumulation pattern of metabolites during mulberry fruit ripening, we conducted targeted metabolomics for absolute quantitative analysis of a number of anthocyanin-related substances. LC-ESI-MS/ MS system (HPLC: Shim-pack UFLC SHIMADZU CBM30A system; MS: Applied Biosystems 6500 Q 
TRAP) was used to analyze extracted samples. LIT and triple quadrupole (QQQ) scans were attained using a triple quadrupole-linear ion trap mass spectrometer $(\mathrm{Q}$ TRAP), and an API 6500 Q TRAP LC/MS/MS system, equipped with an ESI Turbo Ion-Spray interface, operating in the positive ion mode and controlled by Analyst 1.6 software (AB Sciex). With the analytical conditions, the HPLC column used was Waters ACQUITY UPLC HSS T3 C18 $(1.8 \mu \mathrm{m}, 2.1 \mathrm{~mm} \times 100 \mathrm{~mm})$; The mobile phase was composed of two solvents, $0.04 \%$ acetic acid (solvent A): acetonitrile with $0.04 \%$ acetic acid (solvent B). The linear gradient elution program was set as: $100 \%$ A for $0 \mathrm{~min}, 100-5 \%$ A for $12.0 \mathrm{~min}, 5-95 \%$ A for 12.1 min, and maintained at 5-95\% A for $15.0 \mathrm{~min}$. The flow rate was $0.40 \mathrm{~mL} / \mathrm{min}$; temperature was $40{ }^{\circ} \mathrm{C}$ and injection volume was $2 \mu \mathrm{L}$. The eluent was connected to an ESI-triple quadrupole-linear ion trap (Q TRAP)-MS. To ensure reproducibility and reliability, at least three independent biological replicates were analyzed for each sample using the LC-ESI-MS/MS system.

\section{Validation and expression analysis}

A set of genes predicted by RNA sequencing to be related to color development were selected for $\mathrm{qPCR}$ assays. First-strand cDNA was synthesized using a FastQuant RT Kit (TIANGEN Biotech, KR106, Beijing, China). Gene-specific primers for qPCR were designed using Primer Premier software (Table S3). The Morus010170 gene was used as an internal control to normalize gene expression. The qPCR assays were performed using SYBR Premix Ex TaqII (Tli RNaseH Plus; Takara Bio, Shiga, Japan) in a LightCycler 480 instrument (Roche). To ensure reproducibility and reliability, three biological replicates were performed for each gene. We performed regression analysis between the $\mathrm{qPCR}$ and RNA sequencing data for 20 genes of the two genotypes at the three fruit-ripening stages using $\mathrm{R}$ version 3.1.3 (http://cran.r-project.org/).

\section{Statistical analysis}

All the tests were repeated three times. The data was examined via analysis of variance (one-way ANOVA). The statistical analysis was performed using PASW Statistics 18.

\section{Supplementary information}

Supplementary information accompanies this paper at https://doi.org/10. 1186/s12870-020-02486-1.

Additional file 1: Table S1. Transcriptome sequence numbers of mapped reads from the two mulberry genotypes at three developmental stages.

Additional file 2: Table S2. Core genes related to anthocyanin biosynthesis in mulberry fruit.

Additional file 3: Table S3. Primers used for $q P C R$.
Additional file 4: Figure S1. Biosynthesis pathway in mulberry fruit. a. Phenylalanine, tyrosine, and tryptophan biosynthesis. b. Phenylpropanoid biosynthesis. c. Flavonoid biosynthesis. d. Anthocyanin biosynthesis.

Additional file 5: Figure S2. Expression levels of the core genes and transcription factors involved in anthocyanin biosynthesis in mulberry fruit, measured by $\mathrm{qPCR}$.

\section{Acknowledgements}

We would like to thank Editage (www.editage.com) for editing and reviewing this manuscript.

\section{Consent to publication}

Not applicable.

\section{Authors' contributions}

$\mathrm{GQH}, \mathrm{GL}$, and ZZG conceived and designed the experiments. GQH, YCZ, and LW performed the experiments. GQH, YCZ, YQY, and JD analyzed the data. $\mathrm{GQH}, \mathrm{YCZ}$, and ZZG contributed to writing of the manuscript and approved the final manuscript.

\section{Funding}

In this work, the design of the study, collection and analysis was supported by grants from the Promoting the Innovation Ability of Sichuan Province (2016ZYPZ-030), the 13th Five-Year Plan for Crop Breeding Project of Sichuan Province (2016NYZ0041-01) and the Promoting the Financial Innovation Ability of Sichuan Province-Special Project of Youth Fund (2020QNJJ-006). The data interpretation of this study was supported by the Special Project of Applied Technology Research and Development in Nanchong (19YFZJ0061) and the National Modern Agricultural Industry Technology System Sichuan Sericulture Innovation Team (2018CSTD-01). The manuscript writing of this study was supported by the Technology Achievements Conversion Demonstration of Sichuan Province (2019ZHCG0032) and the Key Laboratory of Silkworm and Mulberry Genetic Improvement, Ministry of Agriculture and Rural Affairs (KL201912).

\section{Availability of data and materials}

The datasets generated and/or analyzed during the current study are available at NCBI project PRJNA636910 (https://www.ncbi.nlm.nih.gov/ bioproject/PRJNA636910) with accession number SRP265866.

Any reasonable requests are available from the corresponding author.

Ethics approval and consent to participate

There is no ethics approval and consent to participate in this manuscript.

\section{Competing interests}

The authors declare that they have no conflict of interests.

\section{Author details}

'School of Biotechnology, Jiangsu University of Science and Technology, Zhenjiang 212018, Jiangsu, China. ${ }^{2}$ Sericultural Research Institute, Sichuan Academy of Agricultural Sciences, Nanchong 637000, Sichuan, China.

${ }^{3}$ Sericultural Research Institute, Chinese Academy of Agricultural Sciences, Zhenjiang 212003, Jiangsu, China.

Received: 30 January 2020 Accepted: 9 June 2020 Published online: 17 June 2020

\section{References}

1. Andersen $\varnothing \mathrm{M}$, Jordheim $\mathrm{M}$. The anthocyanins. In flavonoids: chemistry, biochemistry and applications, ed. ØM Andersen, KR Markham, pp. 471-552. Boca Raton, FL: CRC Press; 2006.

2. Kong JM, Chia LS, Goh NK, Chia TF, Brouillard R. Analysis and biological activities of anthocyanins. Phytochem. 2003;64:923-33.

3. Qi, Y. Y, Lou, Q., Li, H.B., Yue, J., Liu, Y.L., \& Wang Y.J. Anatomical and biochemical studies of bicolored flower development in Muscari latifolium. Protoplasma, 250, 1273-1281 (2013).

4. Konczak, l., Zhang, W. Anthocyanins - more than nature's colours. J. Biomed. Biotechnol. 239-240 (2004). 
5. Wrolstad, R.E. Symposium 12: interaction of natural colors with other ingredients. Anthocyanin pigments - Bioactivity and coloring properties J Food Sci 69, C419-C421 (2004).

6. Lee DW, Brammeier S, Smith AP. The selective advantages of anthocyanins in developing leaves of mango and cacao. Biotropica. 1987;19:40-9.

7. He J, Giusti MM. Anthocyanins: natural colorants with health-promoting properties. Annu Rev Food Sci Technol. 2010;1:163-87.

8. Dixon RA, Liu C, Jun JH. Metabolic engineering of anthocyanins and condensed tannins in plants. Curr Opin Biotechnol. 2013;24:329-35.

9. Albert NW, Davies KM, Lewis DH, Zhang H, Montefiori M, Brendolise C, Boase MR, Ngo H, Jameson PE, Schwinn KE. A conserved network of transcriptional activators and repressors regulates anthocyanin pigmentation in eudicots. Plant Cell. 2014;26:962-80.

10. Chaves-Silva S, Santos ALD, Chalfun-Júnior A, Zhao J, Peres LEP, Benedito VA. Understanding the genetic regulation of anthocyanin biosynthesis in plants-tools for breeding purple varieties of fruits and vegetables. Phytochemistry. 2018;153:11-27.

11. Saito R, Fukuta N, Ohmiya A, Itoh Y, Ozeki Y, Kuchitsu K, Nakayama M. Regulation of anthocyanin biosynthesis involved in the formation of marginal picotee petals in Petunia. Plant Sci. 2006;170:828-34.

12. Wu X, Beecher GR, Holden JM, Haytowitz DB, Gebhardt SE, Prior RL. Concentrations of anthocyanins in common foods in the United States and estimation of normal consumption. J Agric Food Chem. 2006:54:4069-75.

13. Li SZ. Compendium of Materia Medica. Beijing: People's Medical Press; 1982. p. 2066-7.

14. Kang TH, Hur JY, Kim HB, Ryu JH, Kim SY. Neuroprotective effects of the cyanidin-3-O- $\beta$ - $d$-glucopyranoside isolated from mulberry fruit against cerebral ischemia. Neurosci Lett. 2006:391:122-6.

15. Lu C, Ji DF, Zhu FR, Zhao AC, Luo GQ, Su C. Chinese mulberry cultivars (in Chinese): Press of Southwest China Normal University; 2017.

16. Yang, G.W., Feng, L.C., Jing, C.J., Yu, M.D., Xiang, Z.H. Analysis of genetic structure variance among mulberry (Morus L.) populations. Seri. Sci. 29, 323329 (2003).

17. Qi XW, Shuai Q, Chen H, Fan L, Zeng QW, He NJ. Cloning and expression analyses of the anthocyanin biosynthetic genes in mulberry plants. Mol Gen Genomics. 2014;289:783-93.

18. Dugo P, Mondello L, Errante G, Zappia G, Dugo G. Identification of anthocyanins in berries by narrow-bore high-performance liquid chromatography with electrospray ionization detection. J. Agr. Food Chem. 2001:49:3987-92.

19. Pelletier M. Characterization of flavonol synthase and leucoanthocyanidin dioxygenase genes in Arabidopsis. Further evidence for differential regulation of "early" and "late" genes. Plant Physiol. 1997;113:1437-45.

20. Mattivi F, Guzzon R, Vrhovsek U, Stefanini M, Velasco R. Metabolite profiling of grape: flavonols and anthocyanins. J Agr Food Chem. 2006;54:7692-702.

21. Yoshida K, Mori M, Kondo T. ChemInform abstract: blue flower color development by Anthocyanins: from chemical structure to cell physiology. ChemInform. 2009:40:884-915.

22. Lou, Q., Liu, Y., Qi, Y., Qi, Y.Y., Jiao, S.Z., Tian, F. F, Jiang, L., \& Wang, Y.J. Transcriptome sequencing and metabolite analysis reveals the role of delphinidin metabolism in flower colour in grape hyacinth. J Exp Bot 65, 3157-3164 (2014),

23. Koes RE, Spelt CE, Elzen PJMV, Mol JNM. Cloning and molecular characterization of the chalcone synthase multigene family of Petunia hybrida. Gene. 1989;81:245-57.

24. Clark ST, Verwoerd WS. A systems approach to identifying correlated gene targets for the loss of colour pigmentation in plants. BMC Bioinformatics. 2011;12:343

25. Castellarin SD, Gaspero GD. Transcriptional control of anthocyanin biosynthetic genes in extreme phenotypes for berry pigmentation of naturally occurring grapevines. BMC Plant Biol. 2007;7:46.

26. Wang KL, Bolitho K, Grafton K, Kortstee A, Karunairetnam S, McGhie TK, Espley RV, Hellens RP, Allan AC. An R2R3MYB transcription factor associated with regulation of the anthocyanin biosynthetic pathway in Rosaceae. BMC Plant Biol. 2010;10:50.

27. Yuan Y, Ma XH, Shi YM, Tang DQ. Isolation and expression analysis of six putative structural genes involved in anthocyanin biosynthesis in Tulipa fosteriana. Sci Hortic. 2013;153:93-102.

28. Deroles, S. Anthocyanin biosynthesis in plant cell cultures: a potential source of natural colourants. - In: Kevin G., Kevin D., Chris W. (ed.): Anthocyanins: Biosynthesis, Functions and Applications. p.107-117, Springer, New York (2009).
29. Almeida, J.R., D’Amico, E., Preuss, A., Carbone, F., de Vos, CH Ric., Deiml, B., Mourgues, F., Perrotta, G., Fischer, T.C., Bovy, A.G., Martens, S., \& Rosati, C. Characterization of major enzymes and genes involved in flavonoid and proanthocyanidin biosynthesis during fruit development in strawberry (Fragariaxananassa). Arch. Biochem. Biophys. 465, 61-71 (2007).

30. Halbwirth H, Puhl I, Haas U, Jesik K, Treutter D, Stich K, Two-Phase J. Flavonoid formation in developing strawberry (Fragariaxananassa) fruit. J Agric Food Chem. 2006;54:1479-85.

31. Kim S, Binzel ML, Yoo KS, Park S, Pike LM. Pink $P$ a new locus responsible for a pink trait in onions Allium cepa resulting from natural mutations of anthocyanidin synthase. Mol Gen Genomics. 2004;272:18-27.

32. Debes, M.A., Arias, M.E., Grellet-Bournonville, C.F., Wulff, A.f., MartinezZamora, M.G., Castagnaro, A.P., \& Diaz-Ricci, J.C. Whitr-fruited Duchesnea indica (Rosaceae) is impaired in ANS gene expression. Amer. J. Bot. 98, 20772083 (2011).

33. Li J, Lu RH, Zhao AC, Wang XL, Liu CY, Zhang QY, Wang XH, Umuhoza D, Jin $X Y, L u C, L i Z G, Y u M D$. Isolation and expression analysis of anthocyanin biosynthetic genes in Morus alba L. Biol Plant. 2014;58:618-26.

34. Yang C, Li D, Liu X, Ji C, Hao L, Zhao X, Li X, Chen C, Cheng Z, Zhu L. OsMYB103L, an R2R3-MYB transcription factor, influences leaf rolling and mechanical strength in rice (Oryza sativa L.). BMC Plant Biol. 2014;14:1-15.

35. Stracke R, Werber M, Weisshaar B. The R2R3-MYB gene family in Arabidopsis thaliana. Curr Opin Plant Biol. 2001;4:447-56.

36. Paz-Ares J, Ghosal D, Wienand U, Peterson $P$, Saedler $H$. The regulatory $c 1$ locus of Zea mays encodes a protein with homology to myb protooncogene products and with structural similarities to transcriptional activators. The EMBO J. 1987;6:3553-8.

37. Erika, C., Sara, Z., Laura, F., Flavia, G., Anita, Z., Linda, A., \& Giovanni Battista T. Functional diversification of grapevine MYB5a and MYB5b in the control of flavonoid biosynthesis in a petunia anthocyanin regulatory mutant. Plant Cell Physiol 3, 517 (2014).

38. Deluc L, Bogs J, Walker AR, Ferrier T, Decendit A, Merillon J. The transcription factor VVMYB5b contributes to the regulation of anthocyanin and proanthocyanidin biosynthesis in developing grape berries. Plant Physiol. 2008;147:2041.

39. Lin-Wang K, Micheletti D, Palmer J, Volz R, Lozano L, Espley R, Allan AC. High temperature reduces apple fruit colour via modulation of the anthocyanin regulatory complex. Plant Cell Envir. 2011;34:1176-90.

40. Ma DW, Reichelt M, Yoshida K, Gershenzon J, Constabel CP. Two R2R3-MYB proteins are broad repressors of flavonoid and phenylpropanoid metabolism in poplar. The Plant J. 2018:96:949-65.

41. Pires N, Dolan L. Early evolution of bHLH proteins in plants. Plant Signal Behav. 2010;5:911-2.

42. Ajaykrishna, P.G.G, Sivakumar, T.R., Jin, C., Li, S.H., Chen, H.J., Weng, Y.J., Yin, J., Jia, J.Q., \& Gui, Z.Z. Antioxidant and hemolysis protective effects of phenolicrich extract from mulberry fruits. Pharmacogn Mag 14, 103-109 (2018).

43. Yin, J., Xin, X.D., Weng, Y.J., \& Gui, Z.Z. Transcriptome-wide analysis reveals the progress of Cordyceps militaris subculture degeneration. PLoS One, https://doi.org/10.1371/journal.pone.0186279 (2017).

44. Li R, Yu C, Li Y, Lam TW, Yiu SM, Kristiansen K, Wang J. SOAP2: an improved ultrafast tool for short read alignment. Bioinformatics. 2009;25:1966-7.

45. Rajkumar AP, Qvist P, Lazarus R, Lescai F, Ju J, Nyegaard M, Mors O, Børglum $A D, L i Q$, Christensen JH. Experimental validation of methods for differential gene expression analysis and sample pooling in RNA-seq. BMC Genomics. 2015;16:548

46. Ernst J, Bar-Joseph Z. STEM: a tool for the analysis of short time series gene expression data. BMC Bioinformatics. 2006:7:191.

\section{Publisher's Note}

Springer Nature remains neutral with regard to jurisdictional claims in published maps and institutional affiliations. 